\title{
Cost-utility analysis of radical nephrectomy versus partial nephrectomy in the management of small renal masses: Adjusting for the burden of ensuing chronic kidney disease
}

\author{
Zachary Klinghoffer, BSc, MD, FRCSC, ${ }^{*}$ Jean-Eric Tarride, MD; ${ }^{\dagger}$ Giacomo Novara, MD, FRSCS, ${ }^{;}$Vincenzo \\ Ficarra, MD, FRSCS,; Anil Kapoor, MD, FRSCS, ${ }^{*}$ Bobby Shayegan, MD, FRSCS, Luis H. Braga, MD, FRSCS*
}

*Division of Urology, McMaster University, Hamilton, ON; 'Department of Clinical Epidemiology and Biostatistics, McMaster University, Hamilton, ON; §Department of Oncological and Surgical Sciences, Urology Clinic, University of Padua, Padua, Italy

See related article on page 114.

Cite as: Can Urol Assoc J 2013;7:108-13. htrp://dx.doi.org/10.5489/cuaj.502

\section{Abstract}

Objectives: We compare the cost-utility of laparoscopic radical nephrectomy (LRN), laparoscopic partial nephrectomy (LPN) and open partial nephrectomy (OPN) in the management of small renal masses (SRMs) when the impact of ensuing chronic kidney disease (CKD) disease is considered.

Methods: We designed a Markov decision analysis model with a 10-year time horizon. Estimates of costs, utilities, complication rates and probabilities of developing CKD were derived from the literature. The base case patient was assumed to be a 65 -year-old patient with a $<4-\mathrm{cm}$ unilateral renal mass, a normal contralateral kidney and a normal preoperative serum creatinine. Univariate and probabilistic sensitivity analyses were conducted to address the uncertainty associated with the study parameters.

Results: OPN was the least costly strategy at \$25 941 USD and generated 7.161 quality-adjusted life years (QALYs) over 10 years. LPN yielded 0.098 additional QALYs at an additional cost of $\$ 888$ for an incremental cost-effectiveness ratio of $\$ 9057$ per QALY, well below a commonly cited willingness-to-pay threshold of $\$ 50000$ per QALY. LRN was more costly and yielded fewer QALYs than OPN and LPN. Sensitivity analyses demonstrated our model to be robust to changes to key parameters. Age had no effect on preferred strategy.

Conclusions: Partial nephrectomy (PN) is the preferred treatment strategy for SRMs. In centres where LPN is not available, OPN remains considerably more cost-effective than LRN. Furthermore, our study demonstrates that there is no age at which PN is not preferred to LRN. Our study provides additional evidence to advocate $\mathrm{PN}$ for the management of all amenable SRMs.

\section{Introduction}

Over the last decade, several authors have challenged the widely held belief that having one functional kidney follow- ing radical nephrectomy was sufficient to avoid long-term adverse renal consequences. Numerous large, retrospective studies have demonstrated that in patients with a small renal mass (SRM), a normal appearing contralateral kidney and a normal preoperative serum creatinine, the incidence of chronic kidney disease (CKD) following radical nephrectomy is up to $65 \%$, while following partial nephrectomy it is less than $20 \%{ }^{1-4}$ CKD is associated with an increased risk of hospitalization, cardiovascular events and death. ${ }^{5}$ However, the effects of CKD following radical or partial nephrectomy are not clear. Despite earlier reports to the contrary, recent retrospective series have suggested that partial nephrectomy is associated with an increased overall survival, especially among younger patients. ${ }^{6,7}$ While the risk of death may be uncertain, patients suffering from CKD have a decreased quality of life and present a considerable financial burden to the healthcare system. ${ }^{8}$

These clinical concerns leave the urologist with the following dilemma: when recommending treatment for a SRM, will patients achieve a superior quality of life from the minimal morbidity of laparoscopic radical nephrectomy (LRN) or from the renal functional preservation of open partial nephrectomy (OPN) or laparoscopic partial nephrectomy (LPN)? To the best of our knowledge, no studies to date have addressed this question. Recent guidelines on the management of SRMs from the American Urological Association and European Association of Urology stress the importance of renal functional preservation in treatment choice and advocate partial nephrectomy as first-line management when technically feasible. Despite these recommendations, national utilization trends from the U.S. indicate that most renal masses are treated with radical nephrectomy. ${ }^{9}$ This suggests that a large proportion of urologists and their patients are selecting radical nephrectomy as their treatment of choice. Furthermore, the cost-effectiveness of these strategies has not been studied to date.

The purpose of this study is to compare the cost-utility ratios of LRN, LPN and OPN in the management of SRMs 
Radical vs. partial nephrectomy in small renal masses

when the impact of ensuing CKD is considered using a Markov decision analysis model. The results will help urologists and health resource managers determine the optimal management of this patient population with respect to cost and quality of life.

\section{Methods}

\section{Model design}

A decision tree was constructed (Fig. 1) using decision analysis software (TreeAge Pro Healthcare 2009, TreeAge Software Inc., Williamstown, MA). Each decision arm progresses to a Markov simulation, a type of model where patients transition through different health states over time. LRN, LPN and OPN were assumed to have equivalent oncological outcomes nearing $98 \%$ cancer-specific survival based on a recent meta-analysis; ${ }^{10}$ therefore, cure rate was not incorporated into our model. Patients were assumed to either experience a complication following surgery or remain entirely complication free during the perioperative period. For the purposes of simplifying model design, all types of complications, excluding development of CKD, were grouped together. Complications were assumed to occur immediately. Patients then enter a Markov cycle (Fig. 2) where they begin in a health state of normal renal function. The time to progress from the start of the decision tree to the Markov cycle was considered immediate and no time or quality-adjusted life years (QALYs) were accrued during this period. Progression to states of CKD, dialysis and death, based on type of nephrectomy, were followed for 10 cycles, with each cycle representing 1 year. The base case patient was assumed to be a 65 -year-old male with a $<4-\mathrm{cm}$ unilateral renal mass, a normal appearing contralateral kidney and a normal preoperative serum creatinine.

\section{Model data sources}

Transition probabilities, cost estimates and utility estimates used in our model are summarized in Table 1.

\section{Probability estimates}

The probabilities of suffering a complication following LRN, LPN and OPN were estimated at 3.4\%, 9.0\% and 6.3\%, respectively, based on a recent meta-analysis. ${ }^{11}$ To ensure our model only reflected the most clinically significant stages of CKD, we restricted our definition of CKD to a glomerular filtration rate $(\mathrm{GFR})<45 \mathrm{~mL} / \mathrm{min} / 1.73 \mathrm{~m}^{2}$. While patients with a GFR of 45 to $90 \mathrm{~mL} / \mathrm{min} / 1.73 \mathrm{~m}^{2}$ are classified as having $\mathrm{CKD}$, their management is far less costly and mortality risk less pronounced than those with a lower GFR. ${ }^{5,8,12}$ The yearly probabilities of developing CKD following radical nephrectomy and partial nephrectomy were estimated at $11.2 \%$ and $1.5 \%$, respectively, based on the largest series of patients undergoing nephrectomy that stratified outcomes by GFR. ${ }^{1}$ The incidence of CKD following LPN, performed by those with expertise, and OPN has been shown to be equivalent. ${ }^{13}$ The yearly probability of progressing from CKD to dialysis was estimated at $0.2 \%$, based on the reported progression of KDOQI (Kidney Disease Outcomes Quality Initiative) Stage 3 (GFR 30-59) to hemodialysis. ${ }^{12}$ Age-related mortality was determined from U.S. Life Tables. ${ }^{14}$ The yearly relative risk of death from CKD, compared to patients with normal renal function, was estimated at 1.8 based on a series of over 1 million ambulatory patients with CKD. ${ }^{5}$ The yearly mortality rate of patients on hemodialysis was estimated at $22.3 \%$ from the United Sates Renal Data System (USRDS) 2010 report. $^{15}$

\section{Cost estimates}

Costs were estimated from a payer perspective. All utilized costs were collected from a U.S. setting and adjusted to 2010 U.S. dollars (USD) using the Bureau of Labor Statistics Consumer Price Index. The cost of LRN, LPN and OPN,

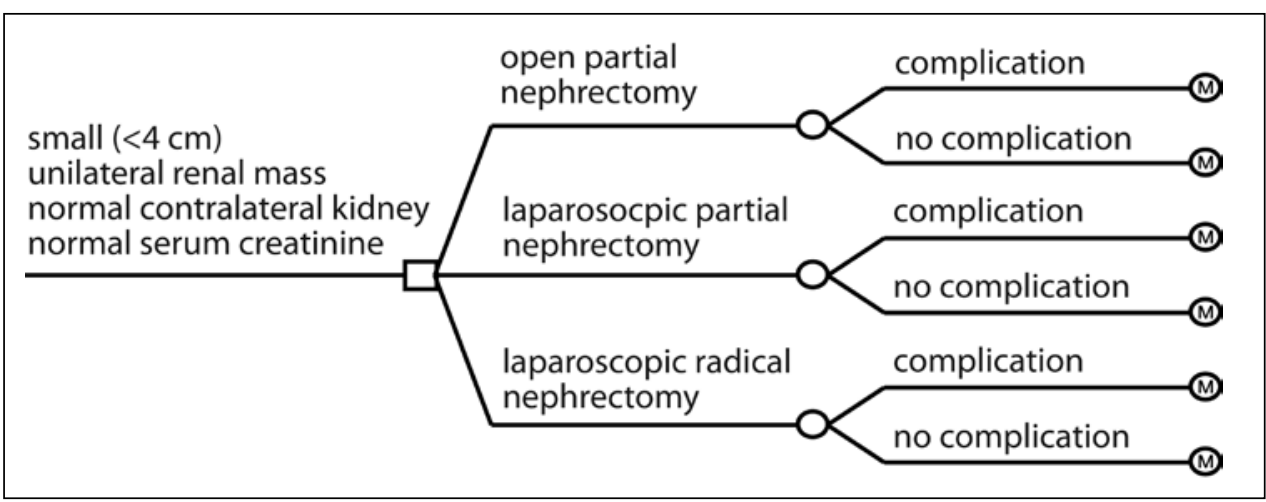

Fig. 1. Decision tree for treatment of a small renal mass. 
Klinghoffer et al.

\begin{tabular}{|c|c|c|c|}
\hline Parameter & Base case estimate & $\begin{array}{c}\begin{array}{c}\text { Univariate sensitivity analysis } \\
\text { range }\end{array} \\
\end{array}$ & Source \\
\hline \multicolumn{4}{|l|}{ Costs } \\
\hline LRN & $\$ 11200$ & $\$ 5000-15000$ & Mouraviev et al., ${ }^{15}$ Bensalah et al. ${ }^{14}$ \\
\hline LPN & $\$ 11657$ & $\$ 5000-15000$ & Mouraviev et al., ${ }^{15}$ Bensalah et al. ${ }^{14}$ \\
\hline OPN & $\$ 11594$ & $\$ 5000-15000$ & Mouraviev et al., ${ }^{15}$ Bensalah et al. ${ }^{14}$ \\
\hline Complication & $3.59 \times$ baseline cost of surgery & $1.5-5.0 \times$ baseline cost of surgery & Dimick et al. ${ }^{16}$ \\
\hline $\begin{array}{l}\text { Post-nephrectomy surveillance } \\
\text { (1 year) }\end{array}$ & $\$ 427$ & $\$ 150-1500$ & $\begin{array}{c}\text { Medicare Part B reimbursement } \\
\text { schedule }\end{array}$ \\
\hline CKD ( 1 year) & $\$ 14392$ & $\$ 1000-40000$ & USRDS 2010 Report $^{29}$ \\
\hline Hemodialysis ( 1 year) & $\$ 75050$ & $\$ 20000-120000$ & USRDS 2010 Report $^{29}$ \\
\hline Discount & $3 \%$ & $1-5 \%$ & Siegel et al. ${ }^{17}$ \\
\hline \multicolumn{4}{|l|}{ Utilities } \\
\hline LRN & 0.730 & $0.600-0.900$ & $\begin{array}{l}\text { Patel et al., }{ }^{18} \text { Jiang et al., }{ }^{19} \\
\text { Harryman et al., }{ }^{20} \text { Novara et al. } .^{21}\end{array}$ \\
\hline LPN & 0.755 & $0.600-0.900$ & Estimated from Novara et al. ${ }^{21}$ \\
\hline OPN & 0.744 & $0.600-0.900$ & Novara et al. ${ }^{21}$ \\
\hline CKD & 0.706 & $0.500-0.900$ & Diaz-Buxo et al. ${ }^{24}$ \\
\hline Hemodialysis & 0.654 & $0.400-0.800$ & Perlman et al. ${ }^{25}$ \\
\hline \multicolumn{4}{|l|}{ Transition probabilities } \\
\hline Age-related mortality (annually) & U.S. life tables & - & Arias $^{30}$ \\
\hline Complication after LRN & $3.4 \%$ & $2.0-5.5 \%$ & Campbell et al. ${ }^{10}$ \\
\hline Complication after LPN & $9.0 \%$ & $7.7-10.6 \%$ & Campbell et al. ${ }^{10}$ \\
\hline Complication after OPN & $6.3 \%$ & $4.5-8.7 \%$ & Campbell et al. ${ }^{10}$ \\
\hline $\begin{array}{l}\text { CKD after radical nephrectomy } \\
\text { (annually) }\end{array}$ & $11.2 \%$ & $8.9-13.9 \%$ & Huang et al. ${ }^{1}$ \\
\hline $\begin{array}{l}\text { CKD after partial nephrectomy } \\
\text { (annually) }\end{array}$ & $1.5 \%$ & $0.8-2.8 \%$ & Huang et al. ${ }^{1}$ \\
\hline Relative mortality risk of CKD & 1.8 & $1.7-1.9$ & Go et al. ${ }^{5}$ \\
\hline $\begin{array}{l}\text { Mortality rate on hemodialysis } \\
\text { (annually) }\end{array}$ & $22.3 \%$ & $15-30 \%$ & USRDS 2010 Report $^{29}$ \\
\hline
\end{tabular}

including cost of operating room time, personnel and equipment, hospital stay, diagnostic imaging and pharmacy were obtained from reports by Bensalah and colleagues ${ }^{16}$ and Mouraviev and colleagues. ${ }^{17}$ These were the only studies identified in the literature that simultaneously reported costs for LRN, LPN and OPN, providing uniformity across cost

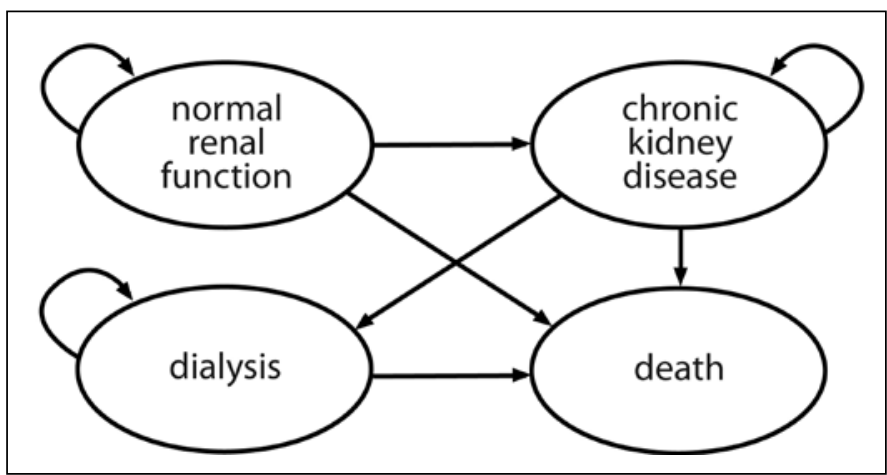

Fig. 2. Health states at each Markov node. Likelihood of progression through each state is determined by the antecedent type of surgery. estimates. The cost of postoperative complications was estimated from Dimick and colleagues. ${ }^{18}$ No consensus exists on appropriate surveillance for patients following radical or partial nephrectomy for renal cell carcinoma. We estimated yearly surveillance costs using 2010 Medicare Part B national reimbursement data for annual computed tomography of the abdomen and pelvis with contrast $(\$ 330)$, chest radiograph (\$31) and physician office visit (\$66) for 10 years. This surveillance strategy is of intermediate to high aggressiveness compared to those reported in the literature. The yearly cost of CKD and hemodialysis was obtained from the USRDS 2010 report. All costs beyond the first year were discounted $3 \%$ annually according to consensus recommendations. ${ }^{19}$ Costs were reported in 2010 USD.

\section{Utility estimates}

QALYs are a commonly used effectiveness measure in decision analysis models. To the best of our knowledge, no studies to date have calculated QALYs following LRN, 
LPN or OPN. Several authors, however, have determined health-related quality of life (HRQOL) using a variety of validated questionnaires, most commonly the Short Form36 Health Survey (SF-36.) A review of studies administering SF-36 questionnaires following LRN, LPN or OPN was performed and authors were contacted for data when SF-36 scores were not reported. ${ }^{20-23}$ SF-36 scores were converted to QALY utility scores using an algorithm developed by Ara and Brazier ${ }^{24}$ and sample-weighted utility scores were then calculated for each type of nephrectomy. The same utility score was used for patients experiencing a complication and for patients who did not experience a complication, as long-term HRQOL following nephrectomy has been shown to be unaffected by complications. ${ }^{25}$ No studies to date have quantified HRQOL following LPN using a validated measure. We estimated the utility of LPN by determining the difference in utility scores between LRN and ORN and adding this value to the utility of OPN. ${ }^{23}$ Utility scores at 1 year were extrapolated across the 10-year time horizon. To maintain uniformity in the HRQOL instruments used to populate our model, we calculated utility scores in a similar fashion for the health states of CKD and dialysis from SF-36 scores reported in the largest published series on HRQOL for those respective health states. ${ }^{26,27}$

\section{Scenario analysis}

Although the incidence of CKD up to 5 years following partial and radical nephrectomy is well-reported, longerterm data is lacking. It is feasible that hyperfiltration injury sustained by a reduced number of nephrons may result in an accelerated rate of development of CKD after a 5-year period. Conversely, the rate of development of CKD may decrease once the acute loss of renal mass due to nephrectomy has been accommodated. We performed a scenario analysis to accommodate this uncertainty. In the first scenario, after 5 years, the rate of development of CKD increased annually by $20 \%$ of the predicted rate. In the second scenario, after 5 years, the rate of development of CKD decreased annually by $20 \%$ of the predicted rate.

\section{Univariate and probabilistic sensitivity analyses}

The robustness of our model to variations in key parameters was first analyzed by performing univariate sensitivity analyses. Each parameter was individually varied across a clinically plausible range of values and the outcome of the model was recalculated throughout this range. The ranges used for each parameter are outlined in Table 1. Although univariate sensitivity analysis can identify the relative influence of individual parameters on model outcome, it inadequately reproduces real-world variability where multiple parameters may change simultaneously.
To address these limitations, we performed a probabilistic sensitivity analysis, which more realistically represents uncertainty by varying each parameter simultaneously. This is achieved by substituting each parameter estimate with a probability distribution and performing a Monte Carlo simulation, where theoretical patients progress through the decision analysis model with parameter values randomly drawn from each probability distribution. We created probability distributions around each parameter using variance reported in the literature. Clinically plausible estimates of variance were used when no published variance data was available. Following standard conventions, costs were modeled with gamma distributions. Transition probabilities and utilities were modeled with beta distributions. The results of 1000 Monte Carlo simulations were plotted on a costeffectiveness axis. A cost-effectiveness acceptability curve (CEAC) was then generated by determining the percentage of simulations that remained cost-effective over a range of willingness-to-pay (WTP) thresholds. The most commonly used WTP threshold, which reflects the highest additional cost stakeholders are willing to pay for one additional QALY, is $\$ 50000$.

\section{Results}

The results of the base case cost-utility analysis are summarized in Table 2. OPN was the least costly strategy at $\$ 25941$ USD and generated 7.161 QALYs over 10 years. Compared to OPN, LPN yielded 0.098 additional QALYs at an additional cost of $\$ 888$. The incremental cost-effectiveness ratio (ICER) of LPN at $\$ 9057$ per QALY made LPN the preferred strategy at a WTP threshold of $\$ 50000$ per QALY. LRN was more costly (\$66 935) and yielded fewer QALYs (6.898) than both OPN and LPN. As such, LRN was dominated by OPN and LPN. The predicted overall survival at 10 years following LPN, OPN and LRN was 93\%, $93 \%$ and $91 \%$, respectively. Freedom from CKD at 10 years following LPN, OPN and LRN was $80 \%, 80 \%$ and $28 \%$, respectively. The percentage of patients on dialysis at 10 years following LPN, OPN, and LRN was $0.07 \%, 0.07 \%$ and $0.4 \%$, respectively.

Univariate sensitivity analysis demonstrated the outcomes of our model to be stable to changes to most parameters. At a WTP threshold of $\$ 50$ 000, OPN replaced LPN

\begin{tabular}{lccc}
\hline \multicolumn{4}{l}{ Table 2. Results of cost-utility analysis } \\
\hline Strategy & Cost (USD) & Utility (QALYs) & ICER \\
\hline OPN & $\$ 25941$ & 7.161 & - \\
LPN* & $\$ 26829$ & 7.259 & $\$ 9057 /$ QALY \\
LRN & $\$ 66935$ & 6.898 & Dominated \\
\hline *Preferred strategy at a willingness-to-pay threshold of $\$ 50$ 000. & \\
USD: U.S. dollar; QALY: quality adjusted life year; ICER: incremental cost-effectiveness \\
ratio; LRN: laparoscopic radical nephrectomy; LPN: laparoscopic partial nephrectomy; OPN: \\
open partial nephrectomy.
\end{tabular}


Klinghoffer et al.

as the preferred strategy under the following conditions alone: (1) if the cost of LPN rose above \$14 913 (compared to $\$ 11657$ in the base case analysis), (2) if the cost of OPN fell below $\$ 8141$ (compared to \$11 594 in the base case analysis), (3) if the utility of LPN fell below 0.746 (compared to 0.755 in the base case analysis, or (4) if the utility of OPN rose above 0.753 (compared to 0.744 in the base case analysis). LRN did not become the preferred strategy under any circumstances. Age had no effect on preferred treatment strategy, though the net monetary benefit of partial nephrectomy over radical nephrectomy diminished with increasing age.

Probabilistic sensitivity analysis reinforced LPN as the preferred strategy. The CEAC showed LPN to have the highest probability of cost-effectiveness across all WTP thresholds.

The relative costs and effectiveness of all strategies remained unchanged in both scenario analyses. LPN remained the preferred strategy at a WTP threshold of $\$ 50000$. When the incidence of CKD increased by $20 \%$ per year after 5 years, the costs of OPN, LPN and LRN increased to $\$ 26585, \$ 27473$ and $\$ 68815$, respectively, while QALYs decreased to 7.158, 7.256 and 6.893, respectively. When the incidence of CKD decreased by $20 \%$ per year after 5 years, the costs of OPN, LPN and LRN decreased to \$25 544, \$26 433 and \$65 580, respectively, while QALYs increased to $7.162,7.261$ and 6.902 , respectively.

\section{Discussion}

Our study demonstrates that partial nephrectomy, either open or laparoscopic, is considerably less costly and yields more QALYs than LRN when accounting for the burden of ensuing CKD. Under no circumstances in our sensitivity or scenario analyses was LRN the preferred treatment modality. The incremental cost of LPN per QALY gained (\$9057/QALY) is far below the commonly cited threshold of $\$ 50000$ per QALY gained and below that of other common interventions, such as screening mammography (\$88 000/ QALY). ${ }^{28}$ In 2010, it was estimated that 58240 new cases of kidney cancer were diagnosed in the United States, of which over 25000 may have been detected as a small, less than $4 \mathrm{~cm}$ renal mass. ${ }^{29}$ It is likely that no more than half of these cases were managed with partial nephrectomy. ${ }^{9}$ Had all cases been treated with OPN or LPN, over 10000 cases of CKD over 10 years could have been averted at a cost savings of almost 1.5 billion USD. In this era of fiscal restraint in healthcare spending, such dramatic savings cannot be ignored.

Our study has a number of limitations. All decision analysis models depend on the quality of data used to populate them. There is a relative paucity of prospective data in the kidney cancer literature, most notably in the realm of health-related HRQOL. Much of our data were extracted from meta-analyses and large prospective or retrospective series. However, many of these were single-institution series. For parameters for which only smaller series were available, our data were enriched by pooling patient level data from contacted authors. Furthermore, the uncertainty of the model parameters was addressed with a comprehensive sensitivity analysis, which reinforced the conclusions from our base case analysis. The ideal method to determine the differences in costs, renal functional outcomes and HRQOL between radical and partial nephrectomy would be a prospective, randomized controlled trial. Unfortunately, the compelling existing evidence supporting the use of partial nephrectomy has limited the feasibility of conducting such a trial as demonstrated by the poor accrual of the prematurely closed EORTC (European Organisation for the Research and Treatment of Cancer) Intergroup study. ${ }^{30}$

Due to a lack of published evidence, we were unable to conduct our cost-utility analysis from a societal perspective according to consensus recommendations. To the best of our knowledge, no studies have measured the costs associated with patient productivity loss, hired caregivers, and outpatient pharmacy following nephrectomy for a SRM. This is an important area of future research.

Bias may have been introduced into our model by our choice to restrict our HRQOL sources to those obtaining data using the SF-36 Health Survey, the most widely used generic instrument for the measurement of HRQOL. We excluded the few studies that used other HRQOL questionnaires. Unfortunately, utility scores obtained using different instruments cannot be reliably pooled. Using the SF-36 alone allowed us to capture the greatest number of patient responses, while facilitating HRQOL comparisons between different health states.

Our model excluded newer management options for SRMs, such as active surveillance, radiofrequency ablation, cryoablation, as well as robot-assisted and laparo-endoscopic single site partial nephrectomy. The long-term oncologic, HRQOL and renal functional outcomes of these treatment modalities have yet to be definitively demonstrated and including them in our model design would have required the use of immature or absent data sets. The impact of active surveillance and thermal ablative therapies at older ages will be of particular interest as will the financial implications of increasing use of robot-assisted partial nephrectomy. We anxiously await published renal function outcomes and HRQOL data from these patient populations.

\section{Conclusion}

Partial nephrectomy, either open or laparoscopic, is considerably less costly and yields more QALYs than LRN when accounting for the burden of ensuing CKD. In our attempts to minimize patient morbidity with widespread use of LRN, 
we may have been inadvertently subjecting patients to an iatrogenic chronic disease and its associated costs. These considerations must remain towards the forefront of our minds, second only to oncologic control, when facing the increasingly common dilemma that is the management of the SRM

Competing interests: None declared.

This paper has been peer-reviewed.

\section{References}

1. Huang WC, Levey AS, Serio AM, et al. Chronic kidney disease after nephrectomy in patients with renal cortical tumours: a retrospective cohort study. Lancet Oncol 2006;7:735-40. http://dx.doi.org/10.1016/ S1470-2045(06)70803-8

2. Zorn $\mathrm{KC}$, Gong $E M$, Orvieto $M A$, et al. Comparison of laparoscopic radical and partial nephrectomy: effects on long-term serum creatinine. Urology 2007;69:1035-40. http://dx.doi.org/10.1016/i.urology.2007.01.092

3. Malcolm JB, Bagrodia A, Derweesh IH, et al. Comparison of rates and risk factors for developing chronic renal insufficiency, proteinuria and metabolic acidosis after radical or partial nephrectomy. BJU Int 2009;104:476-81. http://dx.doi.org/10.1111/i.1464-410X.2009.08376.x

4. Jeon $H G$, Jeong IG, Lee JW, et al. Prognostic factors for chronic kidney disease after curative surgery in patients with small renal tumors. Urology 2009;74:1064-8. http://dx.doi.org/10.1016/i.urology.2009.05.090

5. Go AS, Chertow GM, Fan D, et al. Chronic kidney disease and the risks of death, cardiovascular events, and hospitalization. N Engl J Med 2004;351:1296-305. http://dx.doi.org/10.1056/NEJMoa041031

6. Huang WC, Elkin EB, Levey AS, et al. Partial nephrectomy versus radical nephrectomy in patients with small renal tumors-is there a difference in mortality and cardiovascular outcomes? J Urol 2009;181:55-61; discussion 61-2. http://dx.doi.org/10.1016/i.juro.2008.09.017

7. Zini L, Perrotte P, Capitanio U, et al. Radical versus partial nephrectomy: effect on overall and noncancer mortality. Cancer 2009;115:1465-71. http://dx.doi.org/10.1002/cncr.24035

8. Smith DH, Gullion CM, Nichols $G$, et al. Cost of medical care for chronic kidney disease and comorbidity among enrollees in a large HMO population. J Am Soc Nephrol 2004;15:1300-6. http://dx.doi. org/10.1097/01.ASN.0000125670.64996.BB

9. Dulabon $L M$, Lowrance WT, Russo $P$, et al. Trends in renal tumor surgery delivery within the United States. Cancer 2010:1-6.

10. Campbell SC, Novick AC, Belldegrun A, et al. Guideline for management of the clinical T1 renal mass. J Urol 2009;182:1271-9. http://dx.doi.org/10.1016/i.juro.2009.07.004

11. Novick C. Guideline for Management of the Clinical Stage 7 Renal Mass. American Urological Association Education and Research Inc.; 2009.

12. Keith DS, Nichols $G A$, Gullion $C M$, et al. Longitudinal follow-up and outcomes among a population with chronic kidney disease in a large managed care organization. Arch Intern Med 2004;164:659-63. http:// dx.doi.org/10.1001/archinte.164.6.659

13. Gill IS, Kavoussi LR, Lane BR, et al. Comparison of 1,800 laparoscopic and open partial nephrectomies for single renal tumors. J Urol 2007;178:41-6. http://dx.doi.org/10.1016/i.juro.2007.03.038

14. Arias E. United States life tables, 2006. Natl Vital Stat Rep 2010;58:1-40.
15. U.S. Renal Data System. USRDS 2010 Annual Data Report: Atlas of Chronic Kidney Disease and EndStage Renal Disease in the United States. Bethesda, MD: National Institue of Diabetes and Digestive and Kidney Diseases; 2010.

16. Bensalah K, Raman JD, Bagrodia A, et al. Does obesity impact the costs of partial and radical nephrectomy? J Urol 2008;179:1714-7; discussion 1717-8.

17. Mouraviev V, Nosnik I, Robertson C, et al. Comparative financial analysis of minimally invasive surgery to open surgery for small renal tumours $<0 \mathrm{r}=3.5 \mathrm{~cm}$ : a single institutional experience. Eur Urol 2007;51:715-20; discussion 720-1. http://dx.doi.org/10.1016/i.eururo.2006.06.050

18. Dimick JB, Chen SL, Taheri PA, et al. Hospital costs associated with surgical complications: a report from the private-sector National Surgical Quality Improvement Program. J Am Coll Surg 2004;199:531-7. http://dx.doi.org/10.1016/i.jamcollsurg.2004.05.276

19. Siegel JE, Weinstein MC, Russell LB, et al. Recommendations for reporting cost-effectiveness analyses. Panel on Cost-Effectiveness in Health and Medicine. JAMA 1996;276:1339-41. http://dx.doi. org/10.1001/jama.276.16.1339

20. Patel A, Wilson L, Blick C, et al. Health-related quality of life after retroperitoneoscopic and hand-assisted laparoscopic nephrectomy. J Endouro/ 2005;19:849-52. http://dx.doi.org/10.1089/end.2005.19.849

21. Jiang J, Zheng X, Qin J, et al. Health-related quality of life after hand-assisted laparoscopic and open radical nephrectomies of renal cell carcinoma. Int Urol Nephrol 2009;41:23-7. hitp://dx.doi.org/10.1007/ s1 1255-008-9429-5

22. Harryman $\mathrm{OA}$, Davenport $\mathrm{K}$, Keoghane $\mathrm{S}$, et al. A comparative study of quality of life issues relating to open versus laparoscopic nephrectomy: a prospective pragmatic study. J Urol 2009;181:998-1003; discussion 1003. http://dx.doi.org/10.1016/i.juro.2008.11.028

23. Novara $G$, Secco $S$, Botteri $M$, et al. Factors predicting health-related quality of life recovery in patients undergoing surgical treatment for renal tumors: prospective evaluation using the RAND SF-36 Health Survey. Eur Urol 2010;57:112-20. http://dx.doi.org/10.1016/i.eururo.2009.04.023

24. Ara R, Brazier J. Predicting the Short Form-6D Preference-Based Index Using the Eight Mean Short Form-36 Health Dimension Scores: Estimating Preference-Based Health-Related Utilities When Patient Level Data Are not Available. Value Health 2009;12:346-53. http://dx.doi.org/10.1111/i.15244733.2008.00428.x

25. Gratzke C, Seitz M, Bayrle F, et al. Quality of life and perioperative outcomes after retroperitoneoscopic radical nephrectomy (RN), open RN and nephron-sparing surgery in patients with renal cell carcinoma. BJU Int 2009;104:470-5. http://dx.doi.org/10.1111/i.1464-410X.2009.08439.x

26. Diaz-Buxo JA, Lowrie EG, Lew NL, et al. Quality-of-life evaluation using Short Form 36: comparison in hemodialysis and peritoneal dialysis patients. Am J Kidney Dis 2000;35:293-300. http://dx.doi. org/10.1016/S0272-6386(00)70339-8

27. Perlman RL, Finkelstein F0, Liu L, et al. Quality of life in chronic kidney disease (CKD): a cross-sectional analysis in the Renal Research Institute-CKD study. Am J Kidney Dis 2005;45:658-66. http://dx.doi. org/10.1053/i.ajkd.2004.12.021

28. Mandeblatt J, Saha S, Teutsch S, et al. The cost-effectiveness of screening mammography beyond age 65 years: a systematic review for the U.S. Preventive Services Task Force. Ann Intern Med 2003; 139:835-42.

29. Jemal A, Siegel R, Xu J, et al. Cancer statistics, 2010. CA Cancer J Clin 2010;60:277-300. http:// dx.doi.org/10.3322/caac.20073

30. Van Poppel H, Da Pozzo L, Albrecht W, et al. A Prospective, Randomised EORTC Intergroup Phase 3 Study Comparing the Oncologic Outcome of Elective Nephron-Sparing Surgery and Radical Nephrectomy for Low-Stage Renal Cell Carcinoma. Eur Urol 2011;59:543-52. http://dx.doi.org/10.1016/i. eururo.2010.12.013

Correspondence: Dr. Zachary Klinghoffer, McMaster Institute of Urology, 50 Charleton Ave. East, Hamilton, 0N L8N 4A6; fax: 905-836-9038; zachary.klinghoffer@medportal.ca 